\title{
The Iranian fauna of the subfamilies Acaenitinae, Banchinae, Campopleginae, Ophioninae and Tryphoninae (Hymenoptera: Ichneumonidae) with some new records
}

\author{
Ashkan Masnadi-Yazdinejad, Reijo Jussila \& Matthias Riedel
}

\begin{abstract}
Masnadi-Yazdinejad, A., Jussila, R. \& Riedel, M. 2010: The Iranian fauna of the subfamilies Acaenitinae, Banchinae, Campopleginae, Ophioninae and Tryphoninae (Hymenoptera: Ichneumonidae) with some new records. — Entomol. Fennica 21: $70-83$.
\end{abstract}

This paper provides faunistic data for 24 species examined, belonging to 5 ichneumonid subfamilies: Acaentinae (6 species), Banchinae (4 species), Campopleginae (16 species), Ophioninae (4 species), and Tryphoninae (4 species). Of these, 6 species and 3 genera (Arotes, Coleocentrus, Procinetus) and 1 tribe (Coleocentrini) from Acaentinae, 4 species and 2 genera (Arenetra and Exetastes) from Banchinae, 7 species and 1 genus (Rhimphoctona) from Campopleginae, 4 species from Ophioninae and 4 species of Tryphoninae are new records for the Iranian ichneumonid fauna. Previously recorded species are also included. Distributional maps are given for all records. This study increases the number of known Iranian ichneumonids of Acaentinae to 7, Banchinae to 7, Campopleginae to 26, Ophioninae to 18, and Tryphoninae to 23 species.

A. Masnadi-Yazdinejad, Insect Taxonomy Research Department, Iranian Research Institute of Plant Protection; P.O.Box:19395-1454, Teheran, Iran; Email: amasnadi@yahoo.com,masnadi@iripp.ir

R. Jussila, Zoological Museum, Centre for Biodiversity, Department of Biology, FI-20014 University of Turku, Finland; E-mail: reijo.jussila@utu.fi

M. Riedel, Klinik Fallingbostel, Kolkweg 1, D-29683 Bad Fallingbostel, Germany; E-mail: mamaflo.riedel@t-online.de

Received 25 June 2007, accepted 16 November 2009

\section{Introduction}

Iran is located in the western Palaearctic region with some Oriental characteristics introduced from the south-east. The distribution and biology of the majority of Iranian ichneumonid wasps are not well known. The hymenopterous family Ichneumonidae with 39 subfamilies and approximately 60,000 known species is one of the largest insect families of the world (Yu et al. 2005).

This paper provides additional records of ichneumonids from Iran, and presents their distribution within the country. It also refers to all previously recorded species from Iran.

The aim of this paper is to improve our understanding of and to provide more information about the fauna and distribution of the ichneumonid wasps from the subfamiles Acaentinae, Banchinae, Campopleginae, Ophioninae and Tryphoninae in Iran.

This paper is the last part in the series of Iranian ichneumonid wasp species which were pre- 
Table 1. The Iranian species of Acaentinae. New records are indicated by an asterisk (*).

\begin{tabular}{ll}
\hline Species References & Refor
\end{tabular}

Acaenitini

${ }^{*}$ Arotes albicinctus Gravenhorst, 1829

Phaenolobus araxicola Kasparyan, 1985

Kolarov \& Ghahari 2006

*Phaenolobus areolator (Constantineanu \& Constantineanu, 1968)

*Phaenolobus fulvicornis (Gravenhorst, 1829)

Coleocentrini

*Coleocentrus caligatus Gravenhorst, 1829

*Coleocentrus croceicornis (Gravenhorst, 1829)

*Procinetus decimator (Gravenhorst, 1829)

Table 2. The Iranian species of Banchinae. New records are indicated by an asterisk $\left(^{*}\right)$.

\begin{tabular}{ll}
\hline Species & References
\end{tabular}

Atrophini

Arenetra agrotidis Kokujev, 1906

Aubert 1978, Kolarov \& Ghahari 2005

${ }^{*}$ Arenetra pilosella (Gravenhorst, 1829)

Lissonota (Loxonota) lineate Gravenhorst, $1829 \quad$ Aubert 1978, Kolarov \& Ghahari 2005

*Lissonota magdalenae Pfankuch, 1921

Banchini

Banchus dilatatorius (Thunberg, 1822)

${ }^{*}$ Exetastes fornicator (Fabricius, 1781)

Fitton 1985, Kolarov \& Ghahari 2005

*Exetastes crassus Gravenhorst, 1829

viously authored by Masnadi-Yazdinejad and Jussila (2008a, b \& 2009).

\section{Material and methods}

The material examined was mainly collected during 1999-2006 from several provinces of Iran. Additionally, material preserved at the Hayk Mirzayand Insect Museum (HMIM) was examined. The major sampling techniques used in this study were sweeping, Malaise trap, and light trap.

Identification was carried out referring to the following references: Meier (1968), Rossem (1969), Townes $(1969,1970)$, Kamath and Gupta (1972), Kasparyan (1974) and Schwarz (1999). However, some of the identifications were carried out or confirmed by the second and third authors. All the presented host data is based on Yu et al. (2005).

The entire known Iranian species of five subfamilies, Acaentinae, Banchinae, Campople- ginae, Ophioninae and Tryphoninae with some new additional records are presented. One asterisk indicates new recorded species and 2 asterisks refer to newly recorded genera. The new tribe is marked with an asterisk. The geographical distributions along with dates and localities are given for all material examined. A short comment is presented for each examined species. The references are provided for each previously recorded species within the tables.

All examined materials are deposited in HMIM, located in the Insect Taxonomy Research Department of the Iranian Research Institute of Plant Protection.

\section{Faunistics}

For the subfamily Acaentinae, 6 species from 2 tribes are listed of which 6 species, 3 genera and one tribe are new records. For the Banchinae, 4 species from 2 tribes are included, of which 4 spe- 
Table 3. The Iranian species of Campopleginae. New records are indicated by an asterisk (*).

\begin{tabular}{|c|c|}
\hline Species & References \\
\hline Alcima pictor Aubert, 1971 & Nikdel et al. 2004, Kolarov \& Ghahari 2005 \\
\hline Bathyplectes anurus (Thomson 1887) & Rowshandel 2000, Kolarov \& Ghahari 2005 \\
\hline Bathyplectes curculionis (Thomson, 1887) & $\begin{array}{l}\text { Saeedi } 2004 \text {, Sabahi et al. 2002, } \\
\text { Kolarov \& Ghahari } 2005\end{array}$ \\
\hline $\begin{array}{l}\text { Campoletis ensator (Gravenhorst, 1829) } \\
{ }^{*} \text { Campoletis mitis (Holmgren, 1860) } \\
{ }^{*} \text { Campoletis rapax (Gravenhorst, 1829) }\end{array}$ & Davachi \& Shojaei 1968 \\
\hline $\begin{array}{l}\text { Campoletis thomsoni (Roman, 1915) } \\
{ }^{*} \text { Campoplex deficiens Gravenhorst, } 1829\end{array}$ & Masnadi-Yazdinejad 2006 \\
\hline Campoplex multicinctus Gravenhorst, 1829 & Masnadi-Yazdinejad 2006 \\
\hline Casinaria tenuiventris (Gravenhorst, 1829) & Herard et al. 1979, Rajabi 1986 \\
\hline Diadegma anurum (Thomson, 1887) & Golizadeh et. al. 2008 \\
\hline *Diadegma armillatum (Gravenhorst, 1829) & \\
\hline *Diadegma areolare (Holmgren, 1860) & \\
\hline *Diadegma crassicorne ( Gravenhorst, 1829) & \\
\hline Diadegma majale (Gravenhorst, 1829) & Masnadi-Yazdinejad 2006 \\
\hline Diadegma semiclausum (Hellen, 1949) & Bagheri et al. 2004, Kolarov \& Ghahari 2005 \\
\hline Enytus apostata (Gravenhorst, 1829) & Sooudi et al. 2006 \\
\hline Hellwigia elegans flava Hedwig, 1957 & $\begin{array}{l}\text { Horstmann 1969, Yu \& Horstmann } 1997, \\
\text { Kolarov \& Ghahari } 2005\end{array}$ \\
\hline Hyposoter albipes Hedwig, 1957 & $\begin{array}{l}\text { Horstmann 1969, Yu \& Horstmann } 1997 \\
\text { Kolarov \& Ghahari } 2005\end{array}$ \\
\hline Hyposoter notatus (Gravenhorst, 1829) & Masnadi-Yazdinejad 2006 \\
\hline Leptoperilissus areolaris (Hedwig, 1957) & Yu \& Horstmann 1997, Kolarov \& Ghahari 2005) \\
\hline Leptoperilissus persicus Horstmann, 1993 & Yu \& Horstmann 1997, Kolarov \& Ghahari 2005 \\
\hline $\begin{array}{l}\text { Phobocampe unicincta (Gravenhorst, 1829) } \\
\text { *Rhimphoctona meaacephalus (Gravenhorst 1829) }\end{array}$ & Sedivy 2004, Kolarov \& Ghahari 2005 \\
\hline Sinophorus brunnifemur Sanborne, 1984 & Yu \& Horstmann 1997, Kolarov \& Ghahari 2005 \\
\hline Venturia canescens (Gravenhorst, 1829) & Kolarov \& Ghahari 2005 \\
\hline
\end{tabular}

cies and two genera are new records to Iran. For the subfamily Campopleginae, 23 species are provided including 4 species and 1 genus, which are recorded from Iran for the first time. Out of 18 species belonging to the Ophioninae, 4 species are recorded from Iran for the first time. For the Tryphoninae, 23 species from 2 tribes are included, of which 4 species are new records to Iran.

The complete list of all known species from the five subfamilies is given in Tables $1-5$. It is based both on the material examined by the authors and the literature reviewed. The distributions of all species are presented in Figs. 1-3.

\subsection{Subfamily Acaentinae}

Acaentinae have 240 world-wide and approximately 75 Palaearctic known species (Yu \&
Horstmann 1997), and its diagnostic description is: The labrum is usually conspicuous and semicircular in appearance. The apex of the clypeus is often appearing thick because of its preapical ridge. The protarsal and mesotarsal claws have usually accessory teeth near apex. Apical $0.3-0.5$ of the metasoma is laterally compressed. The female hypogeum is very large and triangular in its lateral view. Relatively few species have been reared; the hosts are larvae in wood or woody tissues (Coleoptera) and probably dubious records of Sesiidae (Lepidoptera) and Siricoidea (Hymenoptera) (Goulet \& Huber 1993).

Only the species Phaenolobus araxicola Kasparyan, 1985 from the tribe Acaentini has been previously recorded from Iran (Kasparyan 1981, Kolarov \& Ghahari 2005). The following list presents the faunistic data, of which, 6 species, 3 genera and 1 tribe are new records for the Iranian Acaentinae. 
Table 4. The Iranian species of Ophioninae. New records are indicated by an asterisk ( $\left.{ }^{*}\right)$.

\begin{tabular}{|c|c|}
\hline Species & References \\
\hline Enicospilus ahngeri Kokujev, 1907 & $\begin{array}{l}\text { Shestakov 1926, Meyer 1935, Hedwig } 1957, \\
\text { Townes et al. 1965, Kolarov \& Ghahari } 2005\end{array}$ \\
\hline Enicospilus cruciator Victorov 1957 & $\begin{array}{l}\text { Kasparyan 1981, Kolarov 1984, Kolarov } \\
\text { \& Ghahari } 2005\end{array}$ \\
\hline Enicospilus ocellatus Shestakov, 1926 & Hedwig 1957, Kolarov \& Ghahari 2005 \\
\hline Enicospilus perlatus Shestakov, 1926 & $\begin{array}{l}\text { Hedwig 1957, Horstmann 1981, Bordera et al. } \\
\text { 1987, Kolarov \& Ghahari } 2006\end{array}$ \\
\hline Enicospilus stenopsis (Kohl, 1905) & $\begin{array}{l}\text { Hedwig 1957, Townes et al. 1965, } \\
\text { Kolarov \& Ghahari } 2006\end{array}$ \\
\hline \multicolumn{2}{|l|}{${ }^{*}$ Enicospilus tournieri (Vollenhoven, 1879) } \\
\hline Enicospilus variicarpus Kokujev 1907 & $\begin{array}{l}\text { Viktorov 1957, Townes et al. 1965, } \\
\text { Kolarov \& Ghahari } 2005\end{array}$ \\
\hline \multicolumn{2}{|l|}{${ }^{*}$ Eremotylus boguschi (Meyer, 1935) } \\
\hline Eremotylus intermedius (Hedwig, 1957) & Horstmann 1981, Yu \& Horstmann 1997 \\
\hline Eremotylus pumilus (Hedwig, 1957) & Horstmann 1981, Yu \& Horstmann 1997 \\
\hline \multicolumn{2}{|l|}{ *Ophion luteus (Linnaeus, 1758) } \\
\hline Ophiom minutes Kriechbaumer, 1879 & Hedwig 1957, Kolarov \& Ghahari 2005 \\
\hline Ophion mirsa (Shestakov, 1926) & Townes et al. 1965, Yu \& Horstmann 1997 \\
\hline Ophion mocsaryi Brauns, 1889 & Hedwig 1957, Kolarov \& Ghahari 2005 \\
\hline \multicolumn{2}{|l|}{ *Ophion obscuratus Fabricius, 1798} \\
\hline Ophion parvulus Kriechbaumer, 1879 & Hedwig 1957, Kolarov \& Ghahari 2005 \\
\hline Ophion turcomanicus Szepligeti, 1905 & $\begin{array}{l}\text { Townes et al. 1965, Sedivy, 1968, } \\
\text { Yu \& Hortstmann 1997, Kolarov \& Ghahari } 2005\end{array}$ \\
\hline Simophion calvus Victorov, 1961 & $\begin{array}{l}\text { Townes et al. 1965, Horstmann 1981, Aubert 1984, } \\
\text { Kolarov \& Ghahari } 2005\end{array}$ \\
\hline
\end{tabular}

\subsubsection{Tribe Acaentini}

**Arotes albicinctus Gravenhorst, 1829

Phaenolobus moiwanus Matsumura, 1912

Sphalerus bifasciatus Kriechbumer, 1878

Material examined. Fars, 1 , Marvdasht,

21.II.2000, A. Masnadi-yazdunejad.

Distribution. Oriental and Palaearctic.

Comments. The genus Arotes Gravenhorst, 1829 and the species $A$. albicinctus are newly recorded from Iran.

*Phaenolobus areolator (Constantineanu \& Constantineanu, 1968)

Moldacoenitus areolator Constantineanu \& Constantineanu, 1968

Material examined. Tehran, 1 , Taleghan, Kalanal 1,800 m a.s.1., 26.VI.1991, Badii.

Distribution. Palaearctic.

Comments. This species is newly recorded from Iran.

*Phaenolobus fulvicornis (Gravenhorst, 1829)
Collyria erythrogaster Lucas, 1849

Material examined. Ardebil, 1 ô, Meshkinshahr, Ilandu 1,800 m a.s.1., 3.VII.1997. M. Mofidi.

Distribution. Western Palaearctic.

Comments. This species is newly recorded from Iran and its major host species are Phytoecia cephalotes and Phytoecia coerulescens (Col. Cerambucidae).

\subsubsection{Tribe * Coleocentrini}

**Coleocentrus caligatus Gravenhorst, 1829

Coleocentrus maximus Rudow, 1881

Material examined. Kerman, 1 , Koohbanan, 25.III.2006, S. Poor-Mohammadi.

Distribution. Western Palaearctic, eastern Palaearctic.

Comments. This is newly record for the tribe Coleocentrini from Iran. Some of the known host species are Callidium aeneum (Col., Cerambycidae), Phymatodes testaceus (Col., Cerambycidae) and Urocerus gigas (Hym., Siricidae). 
Table 5. The Iranian species of Tryphoninae. New records are indicated by an asterisk $\left(^{*}\right)$.

\begin{tabular}{|c|c|}
\hline Species & References \\
\hline \multicolumn{2}{|l|}{ Phytodietini } \\
\hline $\begin{array}{l}\text { Netelia arabs (Strand, 1911) } \\
* \text { Netelia armeniaca Tolkanitz } 1971\end{array}$ & Horstmann 1981, Kolarov \& Ghahari 2006 \\
\hline $\begin{array}{l}\text { *Netelia armeniaca Tolkanitz, } 1971 \\
\text { Netelia cristata (Thomson, 1888) }\end{array}$ & Kolarov \& Ghahari 2006 \\
\hline $\begin{array}{l}\text { Netelia cristata (Thomson, 1888) } \\
\text { Netelia dilatata (Thomson, 1888) }\end{array}$ & Kolarov \& Ghahari 2006 \\
\hline Netelia dilatata (Thomson, 1888) & $\begin{array}{l}\text { Tolkanitz 1981, Kasparyan 1981, Kasparyan \& } \\
\text { Tolkanitz 1999, Kolarov \& Ghahari } 2005\end{array}$ \\
\hline Netelia fuscicornis (Holmgren, 1980) & Kolarov \& Ghahari 2006 \\
\hline \multicolumn{2}{|l|}{${ }^{*}$ Netelia grumi (Kokujev, 1906) } \\
\hline Netelia krishtali Tolkanitz, 1971 & $\begin{array}{l}\text { Kasparyan 1981, Kolarov 1994, Kasparyan \& } \\
\text { Tolkanitz 1999, Kolarov \& Ghahari } 2005\end{array}$ \\
\hline Netelia latungula (Thomson, 1888) & Kolarov \& Ghahari 2006 \\
\hline Netelia lineolata (Costa, 1883) & Kolarov \& Ghahari 2006 \\
\hline Netelia nigricornis Horstmann, 1981 & Yu \& Hortstmann 1997 \\
\hline \multicolumn{2}{|l|}{ “Netelia ocellaris (Thomson, 1888) } \\
\hline Netelia semenovi (Kokujev, 1899) & Townes et al. 1965, Kolarov \& Ghahari, 2005 \\
\hline Netelia testacea (Gravenhorst, 1829) & $\begin{array}{l}\text { Hedwig 1957, Townes et al. 1965, Constantineanu } \\
\text { 1983, Kolarov \& Ghahari } 2005\end{array}$ \\
\hline Netelia thoracica (Woldstedt, 1880) & $\begin{array}{l}\text { Townes et al. 1965, Kasparyan 1981, Kasparyan \& } \\
\text { Tolkanitz 1999, Kolarov \& Ghahar } 2005\end{array}$ \\
\hline Netelia tunetana (Habermehl, 1923) & Kolarov \& Ghahari 2006 \\
\hline Netelia turanica (Kokujev, 1899) & Kolarov \& Ghahari 2006 \\
\hline Netelia vinulae (Scopoli, 1763) & $\begin{array}{l}\text { Tolkanitz 1981, Kasparyan 1981, Kasparyan \& } \\
\text { Tolkanitz 1999, Kolarov \& Ghahari } 2005\end{array}$ \\
\hline \multicolumn{2}{|l|}{ Tryphonini } \\
\hline Tryphon atriceps Stephans, 1835 & Kasparyan 1973, Kolarov \& Ghahari 2005 \\
\hline \multicolumn{2}{|l|}{ *Tryphon hinzi Heinrich, 1953} \\
\hline Tryphon psilosagator Aubert, 1966 & Kolarov \& Ghahari 2006 \\
\hline Tryphon rutilator (Linnaeus, 1761) & $\begin{array}{l}\text { Kasparyan 1973, Kolarov 1977, Kolarov \& Andoni } \\
\text { 1995, Kolarov et al. 1999, Kolarov \& Ghahari } 2005\end{array}$ \\
\hline Zaglyptus multicolor (Gravenhorst, 1829) & Kolarov \& Ghahari 2006 \\
\hline Zaglyptus varipes (Gravenhorst, 1829) & Kolarov \& Ghahari 2006 \\
\hline
\end{tabular}

* Coleocentrus croceicornis (Gravenhorst, 1829)

Macrus soleatus Gravenhorst, 1829

Material examined. Mazandaran, 1 , Behshar, 13.VII.2000. M. Mofidi \& E. Ebrahimi.

Distribution. Western Palaearctic and eastern Palaearctic.

Comments. This species is newly recorded from Iran.

**Procinetus decimator (Gravenhorst, 1829)

Heterolabis aberrans Kriechbaumer, 1889

Heterolabis crassula Kriechbaumer, 1889

Leptobatus biroi Kiss, 1933

Tryphon maculates Gravenhorst, 1829

Material examined. Kohkiluyeh, Boyerahmad, 1 गे, Yasuj, Tang-e Meymand 1,700 m a.s.1., 23.XI.1998, E. Ghilasian.
Distribution. Western Palaearctic and eastern Palaearctic.

Comments. The genus Procinetus Forster, 1869 and the species $P$. decimator are new records from Iran.

\subsection{Subfamily Banchinae}

Banchinae have 1,500 world-wide and approximately 440 Palaearctic known species (Yu \& Horstmann 1997), and its diagnostic description is: Small to large (fore wing 3-16 mm long). Clypeus convex and often not separated from face by groove, the apical margin often with median point; postpectal carina usually complete; fore wing with areolet open; metasomal segment 1 long and slender, without glymma, with no trace 


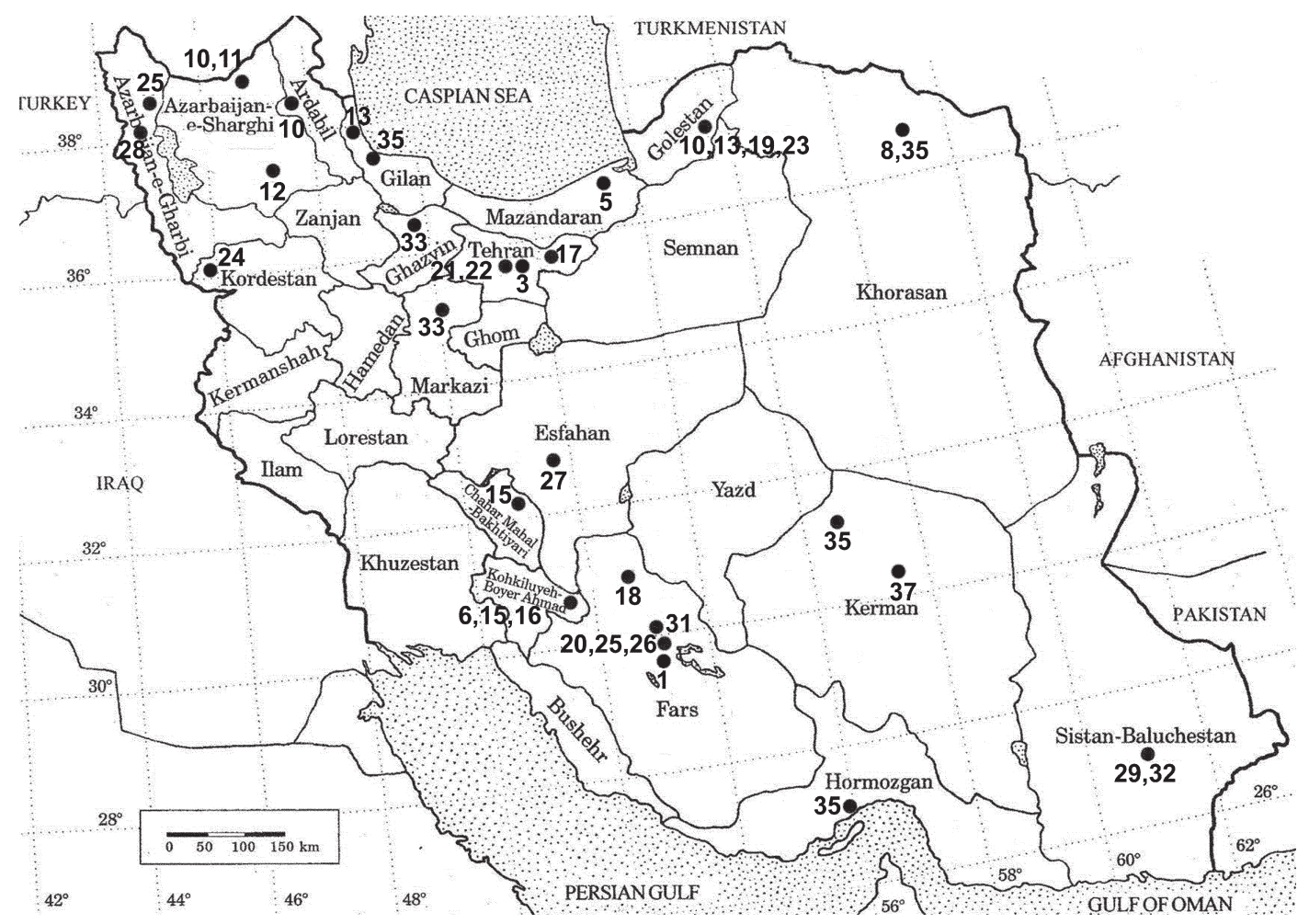

Fig. 1. Distribution of the Iranian species of the subfamilies Acaentinae, Banchinae and Campopleginae. The species with an asterisk has been recorded from Iran without any locality information.

Acaentinae: 1, Arotes albicinctus; 2, Phaenolobus araxicola*;3, Phaenolobus areolator, 4, Coleocentrus caligatus*; 5, Coleocentrus croceicornis; 6 , Procinetus decimator.

Banchinae: 7, Arenetra agrotidis*; 8, Arenetra pilosella; 9, Banchus dilatatorius*; 10, Lissonota lineate; 11, Lissonota magdalenae; 12, Exetastes fornicator, 13, Exetastes crassus.

Campopleginae: 14, Alcima pictor, 15, Bathyplectes anurus; 16, Bathyplectes curculionis; 17, Campoletis ensator, 18, Campoletis mitis; 19, Campoletis rapax; 20, Campoletis thomsoni; 21, Campoplex multicinctus; 22, Casinaria tenuiventris; 23, Diadegma anurum; 24, Diadegma armillatum; 25, Diadegma crassicorne; 26, Diadegma majale; 27, Diadegma semiclausum; 28, Enytus apostate; 29, Hellwigia elegans flava; 30, Hyposoter albipes*; 31, Hyposoter notatus; 32, Leptoperilissus areolaris; 33, Leptoperilissus persicus; 34, Phobocampe unicincta*; 35, Rhimphoctona megacephalus; 36, Sinophorus brunnifemur*; 37, Venturia canescens.

of tergal sternal suture, and with spiracle near apex. This subfamily is known as koinobiont endoparasit of Lepidoptera larvae, parasitizing caterpillars in leaf rolls, tunnels, buds and other concealed situations; however, Banchini parasitize more exposed hosts (specially Noctuidae) (Goulet \& Huber 1993).

Three species belonging to the subfamily Banchinae have been previously recorded from Iran (Kolarov \& Ghahari 2005). The following list provides the faunistic data for 4 new Iranian species from the Atrophini and Banchini.

\subsubsection{Tribe Atrophini}

**Arenetra pilosella (Gravenhorst, 1829)

Arenetra agrotidis, Kokujev, 1906

Material examined. Khorasan 1 o, Mazare-e Astan-e Ghods Unit 2,280 m a.s.1., 18.IV.1997, A. Sarafrazi.

Distribution. Palaearctic.

Comments. The genus Arenetra Holmgren, 1859 and the species $A$. pilosella are newly recorded from Iran. Its host species is Phigalia pilosaria (Lep., Geometridae). 


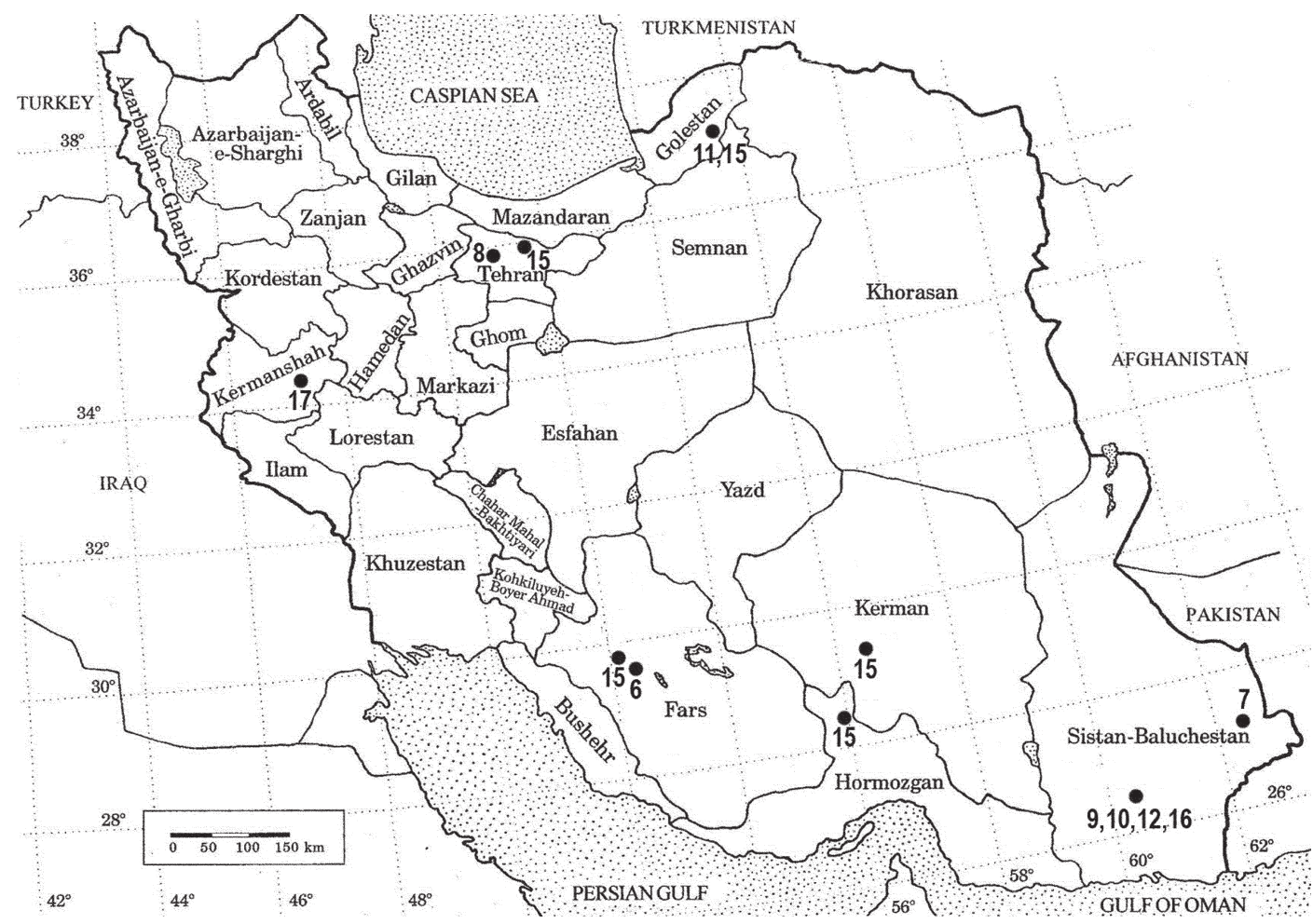

Fig. 2. Distribution of the Iranian species of Ophioninae. The species with an asterisk has been recorded from Iran without any locality information. 1, Enicospilus ahngeri*; 2, Enicospilus cruciator*; 3, Enicospilus ocellatus*; 4, Enicospilus perlatus*; 5, Enicospilus stenopsis*; 6, Enicospilus tournieri; 7, Enicospilus variicarpus; 8 , Eremotylus boguschi; 9, Eremotylus intermedius; 10, Eremotylus pumilus; 11, Ophion luteus; 12, Ophion minutes; 13, Ophion mocsaryi*; 14, Ophion mirsa*; 15, Ophion obscuratus; 16, Ophion parvulus; 17, Ophion turcomanicus*; 18, Simophion calvus*.

* Lissonota magdalenae Pfankuch, 1921

Lissonota vernalis Roman, 1925

Material examined. Ardabil, 1 o, Sabalan, Ghotur, 2,300 m a.s.1., 28.V.1985, H. Mirzayans \& A. Pazuki; Gholestan, 4 우, Park-e Melli-e Golestan, Tangegol, 700 m a.s.1., 25.VIII.1996, E. Ebrahimi; Azarbaijan-e Sharghi, 2 우, Kaleibar, Vayeghan, 1,440 m a.s.1., 5.VIII.1992, M. Parchami-Araghi \& M. Badii.

Distribution. Western Palaearctic.

Comments. This species is newly recorded from Iran.

\subsubsection{Tribe Banchini}

**Exetastes fornicator (Fabricius, 1781) Ichneumon fornicator Fabricius, 1781 Ichneumon expansor Thunberg, 1822 Banchus nervulus Say, 1835
Exetastes punctulatus Kokujev, 1905

Exetastes chosensis Uchida, 1955

Material examined. Azarbaijan-e Sharghi, 1 +, Kaleybar, Vayeghan, 1,440 m a.s.1., 5.VIII.1992, M. Parchami-Araghi \& M. Badii.

Distribution. Palaearctic, Oriental, Nearctic and Neotropical parts.

Comments. The genus Exetastes Gravenhorst, 1829 and the species E. fornicator are new records from Iran. This species is attracted to light and its host species are Abagrotis alternate, Cucullia asteris, Cucullia balsamitae, Cucullia lactucae, Lacanobia oleracea and Mamestra brassicae (Lep., Noctuidae), as well as Mimas tiliae (Lep., Sphingidae), Rhyparia purpurata (Lep., Arctidae) and Trichiosoma sorbi (Hym., Cimbicidae). 


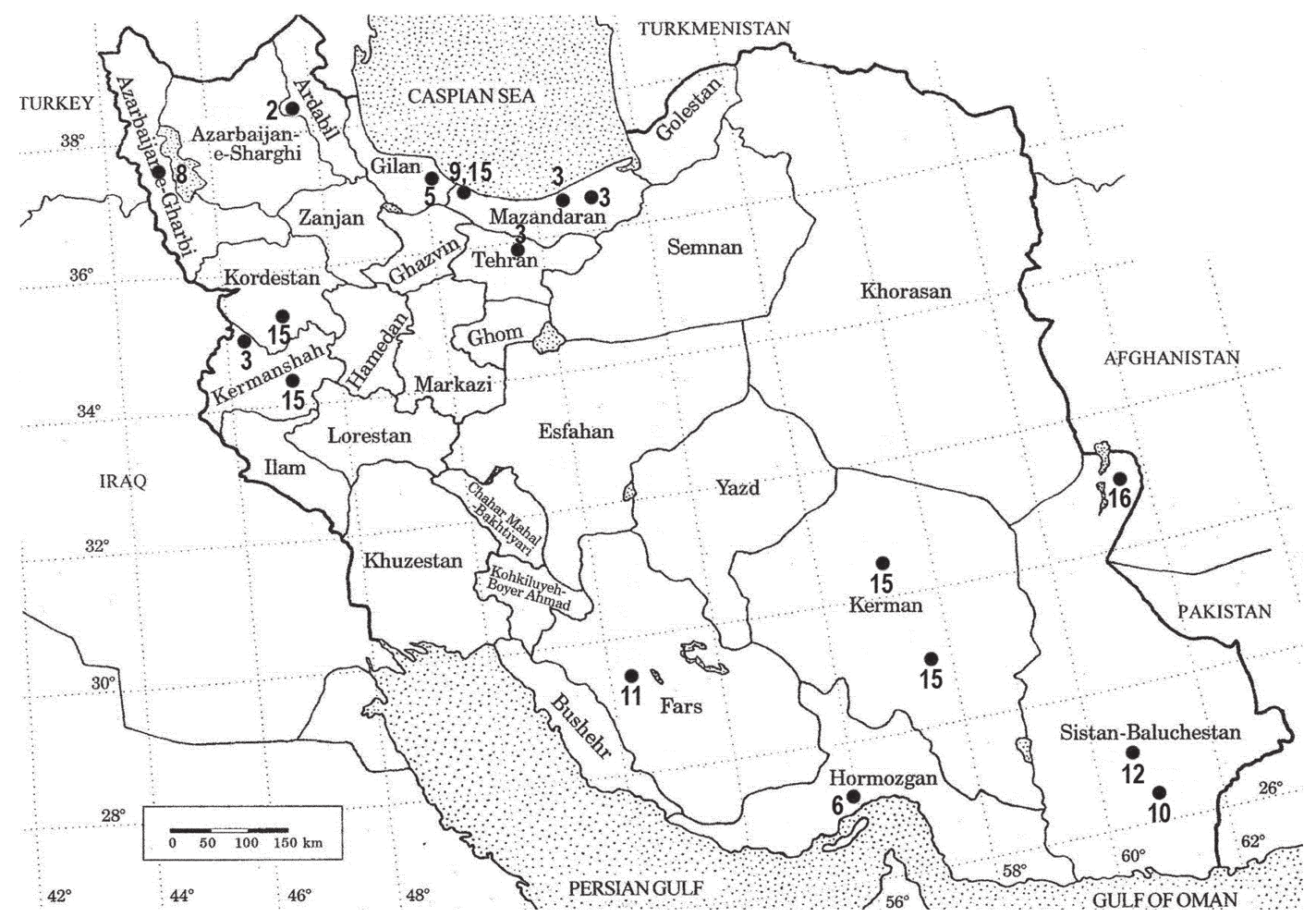

Fig. 3. Distribution of the Iranian species of Tryphoninae. The species with an asterisk has been recorded from Iran without any locality information. 1, Netelia arabs*; 2, Netelia armeniaca; 3, Netelia cristata; 4, Netelia dilatata*; 5, Netelia fuscicornis; 6, Netelia grumi; 7, Netelia krishtali; 8, Netelia latungula; 9, Netelia lineolata; 10, Netelia nigricornis; 11, Netelia ocellaris; 12, Netelia semenovi; 13, Netelia testacea*; 14, Netelia thoracica*; 15, Netelia tunetana; 16, Netelia turanica; 17, Netelia vinulae*.

*Exetastes crassus Gravenhorst, 1829

Exetastes bicoloratus Gravenhorst, 1829

Material examined. Ghilan, 1 , Asalem, 1,250 m a.s.1., 30.VII.1976, H. Bromand \& A. Pazuhi.

Distribution. Palaearctic.

Comments. This species is newly recorded from Iran. Its host species are Colocasia coryli (Lep., Pantheidae), Eligmodonta ziczac (Lep., Notodontidae), Periphanes delphinii, Shargacucullia caninae, Shargacucullia lychnitis and Shargacucullia scrophulariae(Lep., Noctuidae).

\subsection{Subfamily Campopleginae}

Campopleginae has 2,200 world-wide and approximately 1,000 Palaearctic known species (Yu \& Horstmann 1997), and its diagnostic description is: The face of the wasps is usually entirely black; the clypeus is not distinctly separated from the face, and the mandible has a ventral flange; the fore wing has a slender stigma; the ventroposterior corner of the propleuron has a strongly produced lobe touching or overlapping the pronotum; the mesotibial and metatibial spurs are not separated from the first tarsomere by a sclerotized bridge; ovipositor is often upcurved, its dorsal subapical notch is almost always present; the predominant colour is black or black and red; face is rarely pale in the Holarctic species. This subfamily is well known as koinobiont endoparasitoids of Lepidoptera or Symphyta larvae. However, a few of them parasitize Raphididae (Raphidioptera) (Goulet \& Huber 1993). Campopleginae is formerly known as Porizontinae (Townes 1969).

From this subfamily, 18 species have previously been recorded from Iran (Shojaei 1968, Herard et al. 1979, Kasparyan 1981, Kolarov \& 
Ghahari, 2005, Masnadi-Yazdinejad 2006). The following data provide more information for 16 examined species, of which 7 species and 1 genus are new records to Iran.

*Campoletis mitis (Holmgren, 1860)

Sagaritis mitis Holmgren, 1860

Campoplex latrator Gravenhorst, 1829

Sagaritis latratrix Schulz, 1906

Omorgus bicingulatus Szépligeti, 1916

Material examined. Fars, 1 q, Eghlid, Khosroshirin, 2,300 m a.s.1., 22.V.1995, A. Sarafrazi \& M. Badii; 1 + , Neyriz, 1,950 m a.s.1., 4.V.1996, M. Badii.

Distribution. Western Palaearctic, Europe.

Comments. This species is newly recorded from Iran.

Campoletis ensator (Gravenhorst, 1829)

Sagaritis holmgreni Tschek, 1871

Distribution. Eastern and western Palaearctic, Oriental, Iran (Davachi \& Shojaei 1968).

Comments. Some of the important host species are Agrotis segetum (Lep., Noctuidae), Plusia modesta (Lep., Noctuidae) and Yponomeuta plumbella (Lep., Yponomeutidae).

* Campoletis rapax (Gravenhorst, 1829)

Campoples rapax Gravenhorst, 1829

Omorgus curticaudis Szépligeti, 1916

Sagaritis erythropus Thomson, 1887

Material examined. Golestan, 1 q, Park-e Melli Golestan, Dasht-e shad, 1,390 m a.s.1., 25.VII.1996, E. Ebrahimi \& V. Nazari.

Distribution. Eastern and western Palaearctic.

Comments. The species is newly recorded for the Iranian fauna. Some of the important host species are Autographa gamma (Lep., Noctuidae), Lymantria dispar (Lep., Lymantridae) and Ostrinia nubilalis (Lep., Pyralidae).

Campoletis thomsoni (Roman, 1915)

Sagritis thomsoni Roman, 1915

Material examined. Fars, 2 우, Shiraz, 23.VI.1999, A. Masnadi-Yazdinejad; 1 o, Bidezard, 30.VIII.1999. A. Masnadi-Yazdinejad.

Distribution. Western Palaearctic, Iran (Masnadi-Yazdinejad 2006)
*Campoplex deficiens Gravenhorst, 1829

Omorga algerica Hambermehl, 1922

Material examined. Tehran, 1 , Robat Karim, Yagheh, 1,000 m a.s.l., M. Badii.

Distribution. Palaearctic.

Comments. This species is newly recorded from Iran and its important host species is Eupithecia pulchellata (Lep., Geometridae).

Campoplex multicinctus Gravenhorst, 1829

Campoples multicinctus Gravenhorst, 1829

Omorgus excentricus Bauer, 1937

Material examined. Tehran, 1 , Tehran, 1974, Monajjemi.

Distribution. Western Palaearctic, Oriental, Iran (Masnadi-Yazdinejad 2006).

Comments.This species overwinters as larvae. Some of the important host species are Anthonomus pomorum (Col., Curculionidae), Helicoverpa armigera (Lep., Noctuidae) and Pandemis corylana (Lep., Tortricidae).

Casinaria tenuiventris (Gravenhorst, 1829)

Campoples tenuiventris Gravenhorst, 1829

Campoplex conica Ratzeburg, 1844

Casinaria latifrons Holmgren, 1860

Casinaria protensa Thomson, 1887

Material examined. Tehran, 1 गे, Evin, 1986,

M. Abaei \& Gh. Rajabi.

Distribution. Eastern and western Palaearctic, Iran (Herard et al. 1979, Rajabi 1986).

Comments. Some of the important host species are Lymantria dispar (Lep., Lymantridae) and Pieris rapae (Lep., Pieridae).

Diadegma anurum (Thomson, 1887)

Angita anura Thomson, 1889

Material examined. Golestan, 1 + , Parke-e Melli Golestan, Sulgerd, 1,150 m a.s.1., 8.V.1999, H. Barari.

Distribution. Western Palaearctic, Iran (Golizadeh et al. 2008).

Comments. The important host species is Tischeria ekebladella (Lep., Tischeriidae).

* Diadegma areolare (Holmgren, 1860)

Limneria areolaris

Material examined. Azarbayejan-e Sharghi, 1

Oे, Marand, 22.V.2007, H. Lotfalizade.

Distribution. Palaearctic. 
Comments. This species is newly recorded from Iran. The important host species are Cochylis posterana (Lep., Tortricidae), Euclidia glyphica (Lep., Noctuidae).

*Diadegma armillatum (Gravenhorst, 1829)

Angitia monospila Thomson, 1887

Angitia pseudocombinata Szepligeti, 1916

Campoplex tibialis Brischke, 1880

Material examined. Kordestan, 1 , , Bane, 10.VI.1998, A. Masnadi-yazdinejad

Distribution. Palaearctic.

Comments. This species is newly recorded from Iran. The important host species are Agonopterix subpropinquella (Lep., Oecophoridae), Hellula undalis (Lep., Pyralidae), Paraswammerdamia lutarea (Lep., Yponomeutidae), Plutella xylostella (Lep. Plutellidae), Tebenna bjerkandrella (Lep, Choreutidae).

*Diadegma crassicorne (Gravenhorst, 1829)

Campoples crassicornis Gravenhorst, 1829

Campoplex carnifex Gravenhorst, 1829

Limneria noromannica Rudow, 1883

Material examined. Azarbaijan-e-Gharbi, 1 §., Khoy, Habash-e Solfa, 1,825 m a.s.1., 19.VIII.1994, A. Safarrazi \& E. Ebrahimi; Fars, 1 O, Shiraz, Bamu, Parke-e Melli, 1,590 m a.s.1., 14.XI.1997, V. Nazari \& M. Mofidi- Neyestank.

Distribution. Eastern and western Palaearctic.

Comments. This species is newly recorded from Iran. Some of the important host species are Agrotis segetum (Lep., Noctuidae) and Ostrinia nubilalis (Lep., Pyralidae).

\section{Diadegma majale (Gravenhorst, 1829)}

Campoplex majalis Gravenhorst, 1829

Angitia claripennis Thomson, 1887

Material examined. Fars, 2 우, Shiraz, 11.II.1999, A. Masnadi-Yazdinejad; Bajgah, 1 ㅇ and 1 ô, 27.VIII.1999, A. Masnadi-Yazdinejad.

Distribution. Eastern and western Palaearctic, Iran (Masnadi-Yazdinejad 2006).

Comments. Some of the important host species are Plutella xylostella (Lep., Plutellidae), Vanessa atalanta (Lep., Nymphalidae), Yponomeuta malinella and Yponomeuta padella (Lep., Yponomeutidae).
Enytus apostata (Gravenhorst, 1829)

Campoplex apostata Gravenhorst, 1829

Angitia crataegellae Thomson, 1887

Campoplex exareolatus Habermehl, 1922

Horogenes patens Townes, 1945

Material examined. Azarbaija-e Gharbi: 4 우, Kahriz, 6.VIII.2003, Akbarzade.

Distribution. Eastern and western Palaearctic, Ethiopian, Oriental districts and Iran (Sooudi et al. 2006).

Comments. The major host species are Anthonomus pomorum (Col., Curculionidae), Archips rosana (Lep., Tortricidae), Cydia pomonella (Lep., Tortricidae), Ostrinia nubilalis (Lep., Pyralidae), Tortrix viridana (Lep., Tortricidae), Vanessa cardui (Lep., Nymphalidae), Yponomeuta malinella and Yponomeuta padella (Lep., Yponomeutidae).

Hyposoter notatus (Gravenhorst, 1829)

Campoplex notatus Gravenhorst, 1829

Material examined. Fars, 1 , Bajgah, 27.III.2000, A. Masnadi-Yazdinejad; 1 ô, Marvdasht, 3.VI.2000, A. Masnadi-Yazdinejad.

Distribution. Eastern and western Palaearctic, Nearctic, Iran (Masnadi-Yazdinejad 2006).

Comments. Some of the important host species are Agrotis segetum (Lep., Noctuidae), Helicoverpa zea (Lep., Noctuidae) and Polyommatus icarus (Lep., Lycaenidae).

Leptoperilissus areolaris (Hedwig, 1957)

Leptoperilissus areolaris Hedwig, 1957

Nepiesta ocellator Aubert, 1966

Material examined. Kerman, 9 ऽิ $\widehat{\partial}$ and 5 우, Jiroft, Kahnui 5km., 420 m a.s.1., 13.III.1978, A. Pazuki.

Distribution. Eastern and western Palaearctic, Ethiopian, Iran (Hedwig 1957).

**Rhimphoctona megacephalus (Gravenhorst, 1829)

Campoples megacephalus Gravenhorst, 1829

Material examined. Hormozgan, 1 ग, Geno, 1,550 m a.s.1., 19.IV.1994, M. Parchami-Araghi \& E. Ebrahimi.

Distribution. Eastern and western Palaearctic, Oriental.

Comments. The genus Rhimphoctona Foerster, 1869 and the species $R$. megacephalus are new records for the Iranian fauna. 


\subsection{Subfamily Ophioninae}

Ophioninae has 1,200 world-wide and approximately 190 Palaearctic known species (Yu \& Horstmann 1997), and its diagnostic description is: Medium to large specimens (fore wing 6-29 $\mathrm{mm}$ ). The clypeus is separated from the face by a distinct groove, the apical margin has no teeth; the ocelli are always large, with lateral ocelli separated from the eyes by less than their diameter; the antenna has often more than 55 flagellomers. The fore wing has an open areolet, with the vein 3r-m apical to the vein $2 \mathrm{~m}$-cu. The metasomal segment 1 is long, without glymmae and with no trace of tergal-sternal suture; the ovipositor is short, equal to the metasomal height at its apex. This subfamily is well known as koinobiont endoparasitoids of Lepidoptera; nevertheles one species parasitizes Scarabaeidae (Coleoptera) (Goulet \& Huber 1993).

Fourteen species of Ophioninae have previously been recorded from Iran (Kolarov \& Ghahari 2005, Shestakov 1926, Meyer 1935, Hedwig 1957, Viktorov 1957, Townes et al. 1965, Horstmann 1981, Yu \& Horstmann 1997, Šedivy 1968, Morley 1913, Aubert 1984, Kasparyan 1981). The following list provides faunistic data for 4 examined species. All of them are new records for the Iranian fauna.

*Enicospilus tournieri (Vollenhoven, 1879)

Ophion tourneri Vollenhoven, 1879

Enicospilus contributus Shestakov, 1926

Henicospilus rossicus Kokujev, 1907

Material examined. Fars, 1 +, Shiraz, 20.VI.2000, A. Masnadi-Yazdinejad

Distribution. Eastern and western Palaearctic.

Comments. This species is newly recorded for the Iranian fauna and the noctuid host species are Agrotis ipsilon and Agrotis segetum (Lep., Noctuidae).

*Eremotylus boguschi (Meyer, 1935)

Ophion boguschi Meyer, 1935

Clistorapha ventosa Viktorov, 1961

Material examined. Tehran, 1 q, Malard, Khushnam, 25.XII.1991, E. Ebrahimi.

Distribution. Eastern and western Palaearctic, Ethiopian.
Comments. This species is newly recorded for the Iranian fauna.

*Ophion luteus (Linnaeus, 1758)

Ichneumon luteus Linnaeus, 1758

Ichneumon fulvus Fabricius, 1775

Ichneumon vinulae Scopoli, 1763

Ophion calcaratus Morley, 1915

Ophion dispar Branus, 1895

Ophion distans Thomson, 1884

Ophion pictus Habermehl, 1921

Ophion scutellaris Thomson, 1888

Ophion slaviceki Kriechbaumer, 1892

Material examined. Golestan, $5 \circ \circ$, Park-e Melli Golestan, Tang-e Gol, 5.V.1999, H. Barari.

Distribution. Almost worldwide (Australian, eastern and western Palaearctic, Nearctic, Neotropical, Oriental).

Comments. This species is first recorded from Iran and has more than 56 known host species, including Agrotis segetum (Lep., Noctuidae) and Cydia pomonella (Lep., Tortricidae).

*Ophion obscuratus Fabricius, 1798

Ophin obscuratus Fabricius, 1798

Ichneumon polyguttator Thunberg, 1822

Ophion flavopictus Smith, 1874

Material examined. Tehran, $12 \circ \bigcirc$, Lavasan, Naran, 1,650 m a.s.1., 30.IV.1991, E. Ebrahimi \& M. Badii; Golestan, 2 우, Park-e Melli Golestan, Sulgerd 1,150 m a.s.1., H. Barrari \& M. Moghaddam; Hormozgan, 1 q, Haji Abad, 900 m a.s.1., 12.III.1995, A. Sarafrazi \& M. Baddii; Kerman, 1 q, Baft Ghanat-e Marwan, 2,800 m a.s.1., 22.V.1977, M. Safavi; Fars, 1 q Gavkoshak, 10.IV.1976, M. Abaei.

Distribution. Western and eastern Palaearctic.

Comments. This newly recorded species is attracted to light and some of the major host species are Eriogaster lanestris, Malacosoma neustria (Lep., Lasiocampidae) and Noctua fimbriata (Lep., Noctuidae).

\subsection{Subfamily Tryphoninae}

Tryphoninae has 1,300 world-wide and approximately 570 Palaearctic known species (Yu \& Horstmann 1997), and its diagnostic description is: Small to large specimens. The clypeus is large 
and convex, and separated from the face by a groove and its apical margin with a fringe of long parallel setae. The tarsal claws are usually pectinate; the metasomal segment 1 is stout to slender, with the glymma usually present and large. The ovipositor is usually short, not longer than the metasomal height at its apex, without a dorsal subapical notch. The ovipostor has often attached eggs. Most species are ectoparasitoids of Symphyta larvae (Goulet \& Huber 1993). The egg is large and attached to the host by a stalk that is imbedded into the host. Two tribes, Phytodietini and Eclytini attack mainly or only lepidopterous larvae (Townes 1969).

Nineteen species of Tryphoninae have previously been recorded from Iran (Meyer 1931, Townes et al. 1965, Kasparyan 1973, 1981, Horstmann 1981, Tolkanitz 1981, Constantineanu 1983, Kolarov 1994, Kolarov \& Andoni 1995, Kasparyan \& Tolkanitz 1999, Kolarov \& Ghahari 2005, 2006). The following results provide faunistic data for 4 examined species from two tribes that are all presented as new records for the Iranian fauna.

\subsubsection{Tribe Phytodietini}

*Netelia armeniaca Tolkanitz, 1971

Netelia armenica Tolikanitz, 1971

Material examined. Ardabil, 1 ô, Sabalan, Ghotursui, 2,300 m a.s.1., 28.VI.1985, H. Mirzayans \& A. Pazuki.

Distribution. Eastern and western Palaearctic.

Comments. This species is newly recorded for the Iranian fauna.

*Netelia grumi (Kokujev, 1906)

Paniscus grumi Kokujev, 1971

Material examined. Hormozgan, 19 , Geno, 1,550 m a.s.1., 18.IV.1994, M. Parchami-Araghi \& E. Ebrahimi.

Distribution. Eastern and western Palaearctic.

Comments. This species is newly recorded for the Iranian fauna and the host species is Orthosia incerta (Lep., Noctuidae).

* Netelia ocellaris (Thomson, 1888)

Paniscus ocellaris Thomson, 1888

Paniscus longitarsis Cameron, 1899

Material examined. Fars, 1 , , Dashtarjan, 20.VI.1999, A. Masnadi-Yazdinejad.
Distribution. Eastern and western Palaearctic, Oriental.

Comments. This newly recorded species is attracted to light and is known as a koinobiont endoparasitoid of larva of Helicoverpa armigera (Lep., Noctuidae), Lacanobia suasa (Lep., Noctuidae) and Smerinthus ocellatus (Lep., Sphingidae).

\subsubsection{Tribe Tryphonini}

*Tryphon hinzi Heinrich, 1953

Tryphon hinzi Heinrich, 1953

Tryphon clauseni Uchida, 1955

Material examined. Tehran, 1 त, Robat Karim, Yagheh, 1,000 m a.s.1., 19.V.1992, E. Ebrahimi \& M. Badii.

Distribution. Eastern and western Palaearctic.

Comments. This species is the first record of the tribe from Iran.

\section{References}

Aubert, J. F. 1978: Les Ichneumonides ouest-palearctiques et leurs hotes 2. Banchinae et Suppl. aux Pimplinae. Laboratoire d'Evolution des Etres Organises, Paris \& EDIFAT-OPIDA, Echauffour. 318 pp.

Bagheri, M. R., Hatami, B. \& Nematollahi, M. R. 2004: The first record of Diadegma semiclausum (Hellen), endoparasitoiod larvae of Plutella xylostella on Wallflower in greenhouses of Isfahan. - Proceeding $16^{\text {th }}$ Iranian Plant Protection Congress: 162.

Bordera, S., Selfa, J. \& Juminez, R. 1987: Contribution al conocimento del genero Enicospilus Stephens, 1835 (Hym. Ichneumonidae) en Espana. - Boletin de la Asociation Espanola de Entomologia 11: 221-233.

Constantineanu, R. 1983: Ichneumonidae (Hymenoptera) from the "Valea Lui David" Nature reserve (lasi County), new and rate for the Romanian Fauna. - Studii si Cercetari de Biologie Seria Zoologie 35: 77-81. [In Romanian with English summary].

Davachi, A. \& Shojaei, M. 1968: Les Hymenopteres entomophages de I'IRAN-etudes faunestiques - Iranian Plant Protection Congress, October, Tehran. 89 pp.

Fitton, M. G. 1985: The Ichneumon-fly genus Banchus (Hymenoptera) in the Old World. - Bulletin of the British Museum (Natural History), Entomology series 51: 1-60.

Golizadeh, A., Kamali, K., Fathipour, Y., Abbasipour \& Jussila, R. 2008: Report of the Parasitoid wasp, Diadegma anurum (Hym.: Ichneumonidae), from Iran. Journal of Entomological Society of Iran, Vol. 27 supplement: $15-16$.

Goulet, H \& Huber, J. 1993: Hymenoptera of the world. 
An identification guide to families. - Research Branch Agriculture Canada Publication 1894/E. 667 pp.

Hedwig, K. 1957: Ichneumoniden und Braconiden aus Iran 1954. - Jahrbuch Verein vaterländische Naturkunde Württemberg 112: 103-117.

Herard, F., Mercadier, G. \&., Abai, M. 1979: Situation de Lymantria dispar (Lep.: Lymantriidae) et son compexe parasitaire en Iran, ex 1976. - Entomophaga 24: 371-384.

Horstmann, K. 1969: Revision der Hellwigiinae Forster (Hymenoptera, Ichneumonidae). - Mitteilungen der Deutsche Entomologischen Gesellschaft, 28: 30-32.

Horstmann, K. 1981: Insects of Saudi Arabia. Hymenoptera: Fam. Ichneumonidae. - Fauna of Saudi Arabia 3: 425-434.

Kamath, M. K. \& Gupta, V. K. 1972: Ichneumonologia Orientalis Part II. The tribe Rhyssini (Hymenoptera: Ichneumonidae). — Oriental Insects Monograph 2: 1300.

Kasparyan, D. R. 1973: Fauna of the USSR Hymenoptera Vol. III. Number 1. Ichneumonidae (Subfamily Tryphoninae) Tribe Tryphonini. - Nauka publishers, Leningrad: 1-320.

Kasparyan, D. R. 1974: A review of the Palearctic ichneumonids of the tribe Pimplini. The genus Pimpla Fabricius. - Entomologicheskoye Obozreniye 53: 382403. [In Russian.]

Kasparyan, D. R. 1981: A guide to the insects of the European part of the USSR. Hymenoptera, Ichneumonidae. - Opredelitel Fauna SSSR 129: 1-688.

Kasparyan, D. R. \& Tolkanitz, V. I. 1999: Ichneumonidae subfamily Tryphoninae: tribes Sphinctini, Phytodietini, Oedemopsini, Tryphonini (addendum), Idiogrammatini; subfamily Eucerotinae; subfamily Adelognathinae (addendum); subfamily Townesioninae. - Fauna of Russia and neighbouring countries. Insecta Hymenoptera, vol. III, Number 3, New series N143, Nauka, Sankt Petersbourg, 404 pp. [In Russian.]

Kolarov, J. 1977: Tryphoninae (Hymenoptera, Ichneumonidae). Genera and species unknown in Bulgarian fauna up to now. - Travel Science University Plovdiv. 15: $117-126$.

Kolarov, J. 1984: On the species of Bathythrix Foerster (Hymenoptera, Ichneumonidae) in Bulgarian fauna. - Travel Science University Plovdiv 22: 71-76.

Kolarov, J. 1994: Nocturnal Ichneumonidae from Bulgaria and Turkey with description of a new species. - Entomofauna 13: 261-268.

Kolarov, J. \& Andoni, V. 1995: A study of the Albanian Ichneumonidae (Hymenoptera) I. Pimplinae, Tryphoninae and Acaentinae. - Acta Entomologica Bulgarica 2: 28-31.

Kolarov, J., Ozbek, H. \& Yildirim, E. 1999: New distributional date of the Turkish Ichneumonidae (Hymenoptera) I. Pimplinae and Tryphoninae. - Journal of Entomological Research Society 1: 9-15.

Kolarov, J. \& Ghahari, H. 2005: A catalogue of Ichneumonidae (Hymenoptera) from Iran. — Linzer biologische Beitrage 37: 503-532.
Kolarov, J. \& Ghahari, H. 2006: A study of the Iranian Ichneumonidae (Hymenoptera) I. Pimplinae and Tryphoninae. - Zoology in the Middle East 38: 63-68.

Masnadi-Yazdinejad, A. 2006: First report of three genera and species (Hym., Ichneumonidae: Campopleginae) from Iran. - Journal of Entomological Society of Iran 25: 77-78.

Masnadi-Yazdinejad, A. \& Jussila, R. 2008a: A contribution to ichneumonid wasps of Iran (Hym., Ichneumonidae, Diplazontinae, Ichneumoninae and Pimplinae). - Entomofauna, Band 29: 293-320.

Masnadi-Yazdinejad, A. \& Jussila, R. 2008b: A study to the Iranian Cryptinae (Hymenoptera:Ichneumonidae). - Journal of Entomological Society of Iran 28: 1-11.

Masnadi-Yazdinejad, A. \& Jussila, R. 2009: A contribution to ichneumonid wasps of Iran (Hym., Ichneumonidae: Anomaloninae, Cremastinae, Ctenopelmatinae, Mesochorinae, Metopinae and Orthopelmatinae). - Applied Entomology and Phytophatology. Vol. 76: 11-28.

Meier, N. F. 1968: Keys to Parasitic Hymetoptera (Family Ichneumonidae) of the USSR and Adjacent Counries. - Publ. Academy of Seciences ot the USSR. 438 pp.

Meyer, N. F. 1931: Ichneumonidae (Hymenoptera). Trudy Ekspeditsii. - Pamirskaya ekspedoitsiya 1928. 8: 137-141.

Meyer, N. F. 1935: Parasitic Hymenoptera of the family Ichneumonidae of the USSR and adjacent countries. Key to the fauna of the USSR. Part 4. Ophioninae. Leningrad. Akademia Nauk SSSR Press 164: 1-535. [In Russian.]

Morley, C. 1913: The fauna of British India including Ceylon and Burma. Hymenoptera, Vol.3. Ichneumonidae. - British Museum, London. 531pp.

Nikdel, M., Sadeghian, B. \& Dordaei, A. 2004: Report of two ichneumonid species as a parasitoids of brown tail moth (Enproctis chrysorhoea L.) in Araabaran. Proceeding of $16^{\text {th }}$ Iranian Plant protection Congress: 101.

Rajabi, G. H. 1986: Insects attacking rosaceous fruit trees in Iran, Second Volume. Lepidoptera. - Plant Pests and Diseases Research Institute, Tehran Iran. 210 pp.

Rossem, G. van. 1969: A study of the genus Meringopus Förster in Europe and of some related species in Asia. - Tijdschrift voor Entomologie 112: 165-196.

Rowshandel, S. 2000: Biology and efficiency of Bathyplectes anurus in biological control of alfalfa weevil Hyper postica Gyll. - Proceeding of $14^{\text {th }}$ Iranian Plant Protection Congress, September 2000: 28.

Sabahi, Q., Talebi, K. \& Kharrazi, A. 2002: Effects of different doses of four insecticides on mortality and longevity of Bathyplectes curculionis (Thomson) (Hymenoptera: Ichneumonidae) the larval parasitoid of alfalfa weevil Hyper postica Gyll.(Coleoptera: Curculionidae). - Proceeding of $15^{\text {th }}$ Iranian Plant Protection Congress, September 2002: 27.

Shojaei, M. 1968: Entomology, Entomophages. First edition. - Tehran University publications. 464 pp.

Schwarz, M. 1999: Anmerkungen zu einigen westpaläark- 
tischen Arten der Gattung Listrognathus Tschek (Hymenoptera, Ichneumonidae). — Linzer biologische Beiträge 31: 459-463.

Šedivý, J. 1968: Beitrage zur Kenntnis der Fauna Afghanistans. Ichneumonidae, Hym. - Casopis Moravskeho Musea 53 Suplementum: 249-272.

Shestakov A. 1926: Tabula diagnostica et species novae palaearcticae generis Enicospilus Stephen. - Konowia 5: 25-32.

Sooudi, M., Shojai, M., Nasrollahi, A., \& Assar M. 2006: Study of the seasonal fluctuation, damage percentage and determination of pupal parasitoide of Lobesia botrana (Lep.: Tortricidae) in Shahriar region, Ghazvin province(Takestan region). - Proceeding $17^{\text {th }}$ Iranian Plant Protection Congress: 291.

Tolkanitz, V. I. 1981: Parasitic Hymenoptera. Ichneumonidae, Metopiinae. — Fauna Ukraina 11: 148 [In Ukrainian.]

Townes, H., Momoi, S, \& Townes, M. 1965: A catalogue and reclassification of the Eastern Palearctic Ichneumonidae. - Memoirs of the American Entomological Institute 5: 1-661.

Townes, H. 1969: The genera of Ichneumonidae 1. - Memoirs of the American Entomological Institute 11: 1300.

Townes, H. 1970: The genera of Ichneumonidae 2. - Memoirs of the American Entomological Institute 12: 1537.

Yu, D. S. \& Horstmann, K. 1997: A catalogue of world Ichneumonidae (Hymenoptera). - Memoirs of the American Entomological Institute 58. 1558 pp.

Yu, D. S, van Achterberg, K. (Braconidae) \& Horstmann, K. (Ichneumonidae) 2005: World Ichneumonoidea 2004. Taxonomy, Biology, Morphology and Distribution. - CD/DVD. Taxapad, Vancouver, Canada.

Viktorov, G. A. 1957: Species of the genus Enicospilus Stephens in USSR. - Entomologicheskoye Obozreniye 36: 179-210. [In Russian.] 